08

\title{
Влияние электрических полей на процессы самоорганизации в ультрадисперсном растворе многостенных углеродных нанотрубок
}

\author{
(C) А.П. Кузьменко, Тет Пьо Наинг, А.Е. Кузько, Мьо Мин Тан \\ Юго-Западный государственный университет, \\ 305040 Курск, Россия \\ e-mail: apk3527@mail.ru
}

Поступило в Редакцию 11 апреля 2017 г.

В окончательной редакции 14 июня 2018 г.

Принято к публикации 4 августа 2019 г.

Представлены результаты исследований влияния электрического поля на процессы упорядочения в осадках ультрадисперсных растворов из функционализированных многостенных углеродных нанотрубок. Особенности и закономерности процессов самосборки и/или самоорганизации, приводящие к формированию структур в виде линейных, фрактальных и кластерных структур, изучены методами конфокальной (с видеозаписью), атомно-силовой и электронной сканирующей и просвечивающей микроскопий, IR-Фурье спектроскопии, комбинационного (рамановского) рассеяния света и рентгенофазового анализа. Установлено, что размеры фрактальных структур уменьшаются обратно пропорционально напряженности электрического поля, а скорость их роста квадратично возрастает с ростом напряженности. Внутри линейных и кластерных структур обнаружены и охарактеризованы по хиральности одностенные углеродные трубки, обладающие металлической и полупроводникой проводимостями.

Ключевые слова: многостенные углеродные нанотрубки, ультразвуковая функционализация, самосборка и самоорганизация, постоянное электрическое поле, динамика фракталов.

DOI: 10.21883/JTF.2020.02.48821.2009

\section{Введение}

Доступность методов синтеза одностенных (SWCNT) и многостенных (MWCNT) углеродных нанотрубок (CNT), которые, в силу пониженной размерности, проявляют эффекты и свойства квантовой природы, казалось бы, должны были привести к массовой их востребованности в h-tech-областях и, в частности, в нано- и микроэлектронике [1,2]. Однако на этом пути возникло физическое ограничение, обусловленное необычайно высоким уровнем поверхностной энергии CNT, что не только затрудняет манипулирование ими и приводит к структурированию в виде жгутов, клубков, глобул и т.д., но и требует необходимой для их стабилизации либо многостадийной обработки [3], либо введения SWCNT и MWCNT в полимерные [4,5] или металлические матрицы [6,7]. Исследование CNT в настоящее время хорошо обеспечено аппаратно [8-10], в том числе при воздействии как электрических [11-17], так и магнитных полей [4] и различных излучений [18-20]. Наиболее эффективная стабилизация CNT, без которой невозможно их непосредственное применение, в частности для формирования электронных элементов и нанопроводов, достигнута функционализацией карбоксильными группами - СООН [21,22], при которой их агрегирование не наблюдалось 3.5 месяца [23]. Высокая устойчивость углеродных структур, выделенных после разрушения HF кремниевых каркасов природного минерала шунгита, была достигнута после их интенсивной ультразвуковой обработки, что позволило изучить процессы их нанострук- турирования в постоянном электрическом поле [24], доказать возможность формирования на этой основе нанопроводов (патент РФ № 2533330 от 15.04.2013) и дать им качественное объяснение на основе явлений самосборки и самоорганизации $[25,26]$.

В настоящей работе с привлечением наноинструментальных методов изучено влияние электрического поля на процессы самосборки и самоорганизации функционализированных MWCNT с разными концентрациями в растворе деионизированной воды $\left(\mathrm{H}_{2} \mathrm{O}\right)$ после интенсивной ультразвуковой обработки (SDS).

\section{Методы и результаты исследования}

Исследованы MWCNT (производство Nanocyl, Бельгия), синтезированные CCVD-методом с нанокатализаторами на основе Со. Чистота MWCNT была на уровне 90\%. На вставке к рис. 1 представлено их изображение, полученное на сканирующем электронном микроскопе (SEM) (JEOL JSM LV6610, 3 nm). Диаметр MWCNT $d \leq 20 \mathrm{~nm}$, длина $l$ достигала до нескольких $\mu \mathrm{m}$, а аспектное отношение $l / d$ не превышало $10^{3}$.

Функционализация MWCNT проводилась перемешиванием в течение $5 \mathrm{~h}$ в ультразвуковой ванне в кислотной среде $\left(\mathrm{H}_{2} \mathrm{SO}_{4}\right.$ и $\mathrm{HNO}_{3}$ в соотношении $\left.3: 1\right)$, возникающий при этом разогрев был не более $42^{\circ} \mathrm{C}$. Охлажденный раствор в равновесном соотношении разбавлялся $\mathrm{H}_{2} \mathrm{O}$, подвергался фильтрованию, промывке и дегидратации [21-23]. Уровень функционализации 


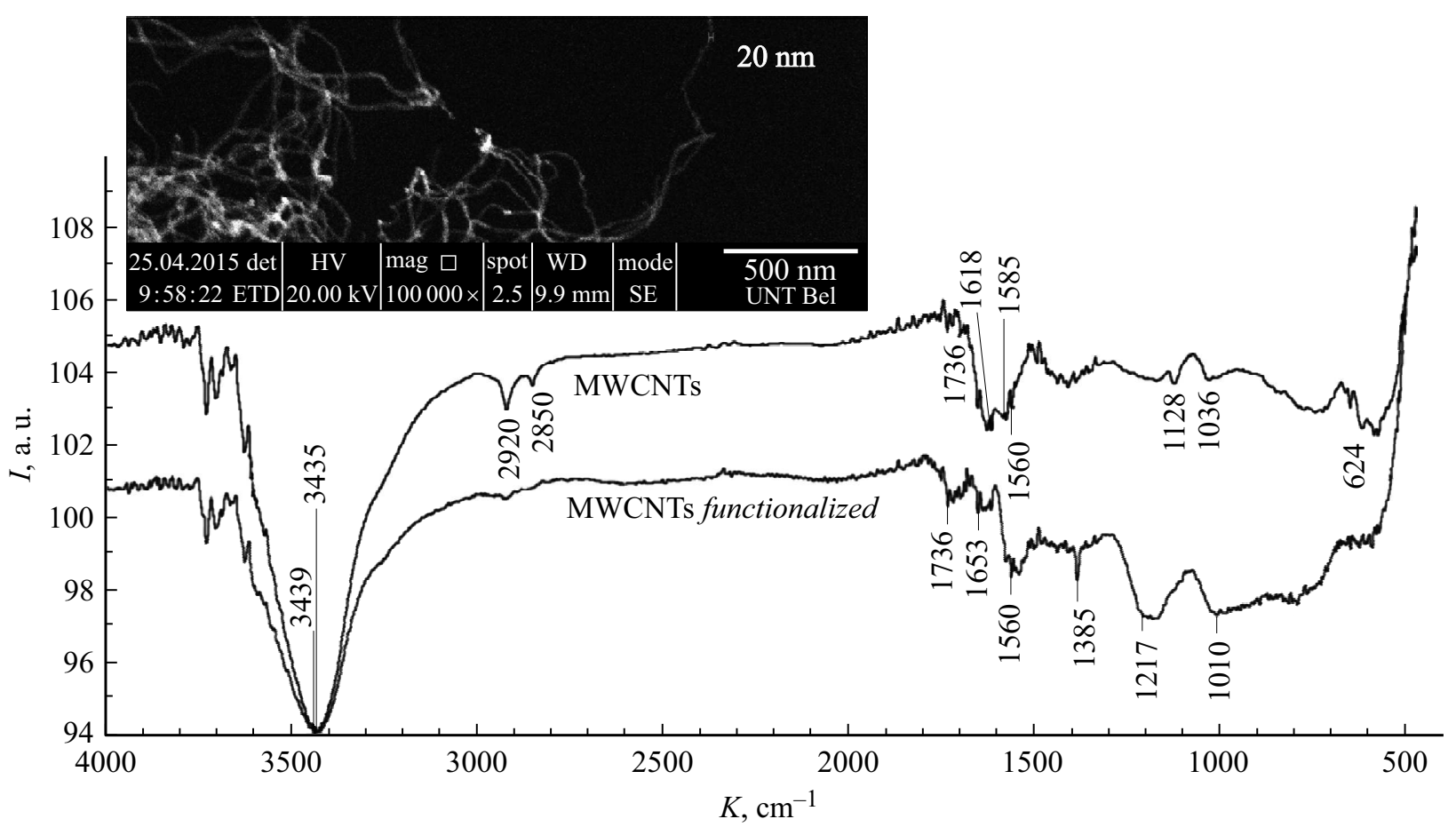

Рис. 1. ИК-Фурье исходных и функционализированных MWCNT. На вставке показано SEM-изображение исходных MWCNT (диаметр $20 \mathrm{~nm}$ ).

MWCNT как в исходном, так и обработанном состоянии в SDS определен по данным IR-Фурье анализа (Nicolet iS50, $0.125 \mathrm{~cm}^{-1}$ ) (рис. 1) в диапазоне измерений (500-4000 cm $\left.\mathrm{cm}^{-1}\right)$ появлением карбоксильной группы в виде несимметричных и симметричных колебаний для $\mathrm{COO}^{-}: v_{\mathrm{as}}-1653$ и $v_{\mathrm{s}}-1385 \mathrm{~cm}^{-1}$ и растягивающих вибрационных $\mathrm{C}-\mathrm{O}-1217 \mathrm{~cm}^{-1}$, а также исчезновением $\mathrm{CH}_{2}$-линий $v_{\mathrm{s}}-2020$ и $v_{\mathrm{s}}-2850 \mathrm{~cm}^{-1}$. Отмечен заметный рост интенсивности и сдвиг отдельных линий валентных колебаний $(\mathrm{O}-\mathrm{H},-\mathrm{OH})-$ $3435 \mathrm{~cm}^{-1}$ на $4 \mathrm{~cm}^{-1}$, растягивающих мод карбоксильных групп $(-\mathrm{C}=\mathrm{O})-1736 \mathrm{~cm}^{-1}$ и ароматического колец $(-\mathrm{C}=\mathrm{C}-)-1618$ на $37 \mathrm{~cm}^{-1}$ и $(-\mathrm{C}=\mathrm{C})-$ $1585 \mathrm{~cm}^{-1}$ на $25 \mathrm{~cm}^{-1}$ и растягивающих вибрационных колебаний $(\mathrm{C}-\mathrm{O})-1036 \mathrm{~cm}^{-1}$ на $8 \mathrm{~cm}^{-1}$. Обнаруженные изменения в IR-спектрах соответствовали функционализации MWCNT и согласовывались с выводами [3,21-23].

Из полученных комплексов MWCNT путем последовательно проводимых ультразвукового перемешивания и диспергирования создавались два типа $\mathrm{SDS}$ - либо MWCNT $+\mathrm{H}_{2} \mathrm{O}$ - SDSI, либо $\mathrm{MWCNT}+\mathrm{NaCl}_{2} \mathrm{H}_{25} \mathrm{SO}_{4}+\mathrm{H}_{2} \mathrm{O}$ - SDSII. По данным конфокальной микроскопии размеры структур в осадках, возникающих после испарения SDS, уменьшались по зависимости, близкой к экспоненциальной: $D=D_{0} \exp (-k t)$, где $D_{0}-$ начальный размер $\sim 3.5 \mu \mathrm{m}$. Здесь $k-$ временная характеристика диспергатора, а $t$ - время ультразвуковой обработки. В SDSII стабиль- ность сохраняется уже в течение почти трех лет в отличие от [23].

В спектрах комбинационного (рамановского) рассеяния света (RS) (OmegaScope ${ }^{\mathrm{TM}}$ рамановский микроспектрометр, $\left.532 \mathrm{~nm}, 0.8 \mathrm{~cm}^{-1}\right)$ как без, так и с электрическим полем были обнаружены линии $D=1336-1353 \mathrm{~cm}^{-1}$ („дефектная мода“ с симметрией $A$-типа) и $G=1567-1600 \mathrm{~cm}^{-1}$ (тангенциальные колебания) с характерным для MWCNT распределением интенсивности $\left(I_{\mathrm{D}}>I_{\mathrm{G}}\right)$, как это можно видеть на рис. 2. Интенсивность $I_{\mathrm{D}}$ линии $D$ для функционализированных MWCNT по сравнению с ее первоначальной величиной возросла, тогда как интенсивность $I_{\mathrm{G}}$ линии $G$ не изменялась. Отношение $I_{\mathrm{D}} / I_{\mathrm{G}}$, характеризующее дефектность структур MWCNT, в исходном состоянии $\sim 1.2$, а после функционализации выросло до $\sim 1.4$. Рост дефектности MWCNT, очевидно, обусловлен интенсивной ультразвуковой обработкой. Полное время ультразвукового диспергирования доходило до $6 \mathrm{~h}$, что превышало обычно применяемое [21-23]. Можно предположить, что рост времени стабильного существования растворов MWCNT в наших условиях обусловлен именно этим обстоятельством.

Приготовленные SDSI и SDSII наносились методом из капли (объем не более $1 \mu \mathrm{l}$ ) в межэлектродный промежуток $(L=100,500,1000$ и $1500 \mu \mathrm{m})$ печатной платы, изготовленной методом фотолитографии, на подложках из ситалла, кварца или монокристаллического кремния. Пленочные электроды формировались методом магнетронного распыления из $\mathrm{Cu}, \mathrm{Cr}$ или $\mathrm{Au}[24,26]$. 


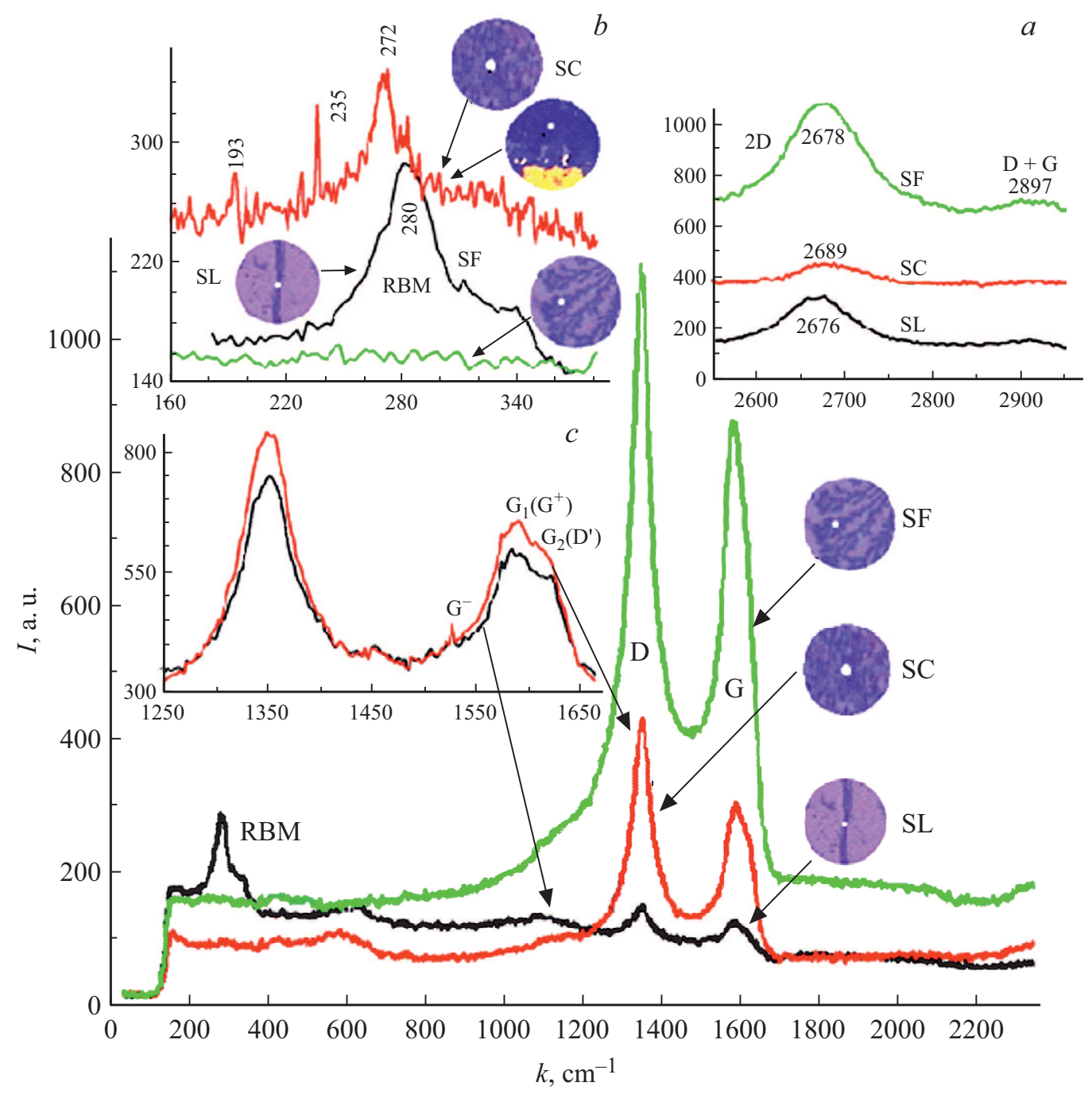

Рис. 2. Комбинационное рассеяние света на линейных образованиях (SL), фрактальных кластерах (SC) и фрактальных структурах $(\mathrm{SF})$, образованных из MWCNT. RS на частотах выше $2200 \mathrm{~cm}^{-1}$ (a) для структур SF, SC, SL, в RBM области $(b)$ с фотографиями структур, на линиях $G^{-}, G_{1}\left(G^{+}\right), G_{2}\left(D^{\prime}\right)(c)$.

На электроды от стабилизированного источника питания (АТН-1351) подавалось постоянное напряжение - $U$, варьируемое от 10 до $50 \mathrm{~V}$, что позволяло варьировать напряженность электрического поля $E=U / L$ в широких пределах — от $7 \cdot 10^{3}$ до $5 \cdot 10^{5} \mathrm{~V} / \mathrm{m}$. Для $L=1500 \mu \mathrm{m}$ и начальной концентрации SDS в диапазоне $0.2-0.8 \mathrm{mg} / \mathrm{ml}$ исходная высота капли $\left(h_{0}\right)$ составляла $500 \mu \mathrm{m}$, а время испарения капли по визуальному контролю составило $t=180 \mathrm{~s}$. Процесс образования нано- и микроструктур начинался после $5 \mathrm{~s}$. Остаточная высота составила $120 \mu \mathrm{m}$. В линейном приближении расчетная скорость испарения капли составила: $V=\left(h-h_{0}\right) /\left(t-t_{0}\right) \sim 2.2 \mu \mathrm{m} / \mathrm{s}$.

Типичные изображения микро- и наноструктур, возникающих в электрическом поле $(E=U / L)$ при испарении капли SDS, были получены с помощью конфокального микроскопа (рис. $3, a, b)$, SEM (рис. 3, c,d) и атомносилового микроскопа (AFM) (AIST-NT) (рис. 4, $a-c$ ).
Движение частиц в SDS обоих типов в электрическом поле в процессе испарения анализировалось по видеозаписям $(30 \mathrm{~Hz}, 704 \times 576 \mathrm{px})$ по аналогии с [24,25]. Катод и анод обозначены „-““ и „+““. Расстояние между ними $1500 \mu \mathrm{m}$. В отсутствие поля MWCNT хаотично агрегировались, на краях капли из них возникали пиннинговые границы из кластеров, а в центральной области формировались фрагменты линейных и фрактальных структур, показанные на вставке к рис. 3, $a, c$. Ситуация изменялась под воздействием поля. Рост структур из MWCNT был строго сориентированным от „-““ к „,+“. Упорядочение по полю можно видеть на конфокальном и SEM-изображениях (рис. $3, b-U=21 \mathrm{~V}$ и $d-$ $U=21 \mathrm{~V})$. Направленный рост линейных и фрактальных структур из MWCNT был изучен по видеозаписям, как это демонстрирует фрейм реально-временного видеоизображения на 13-й s (рис. 3, b). После завершения процесса на 180-й s в центре капли возникали структуры, 
$a$
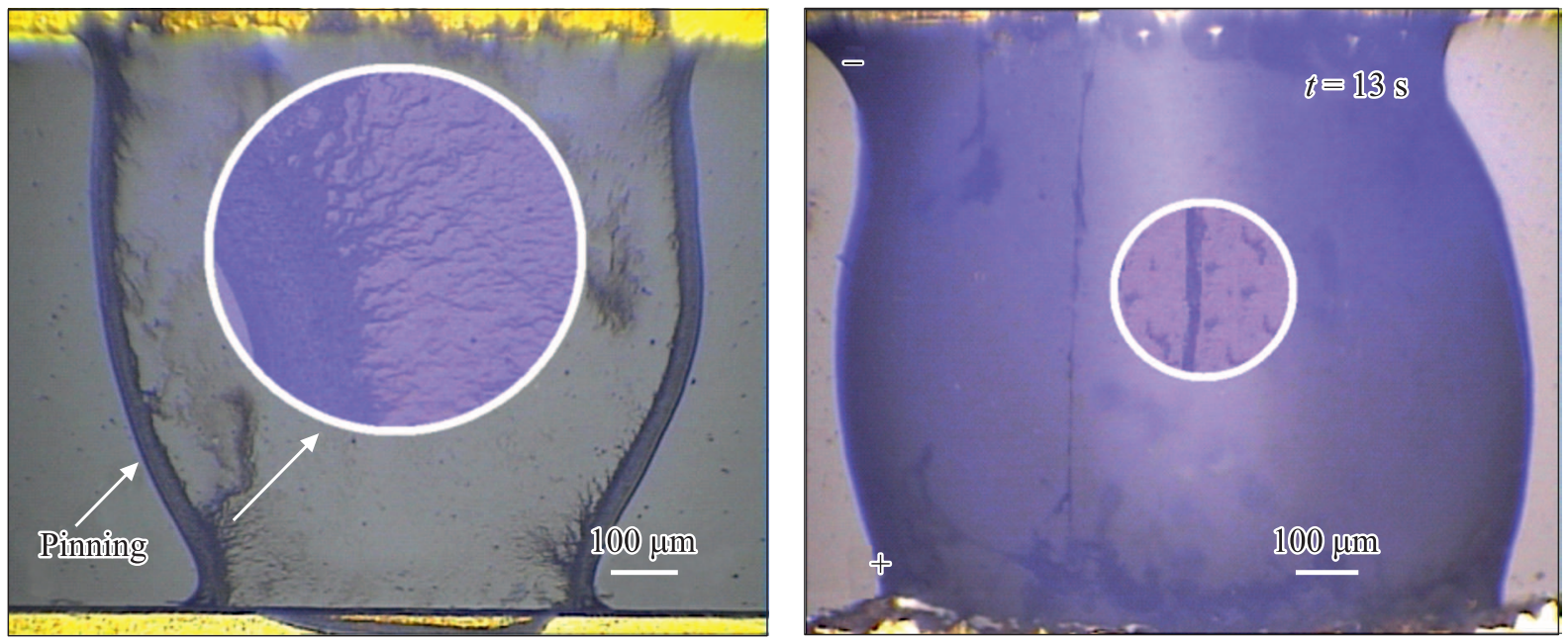

C
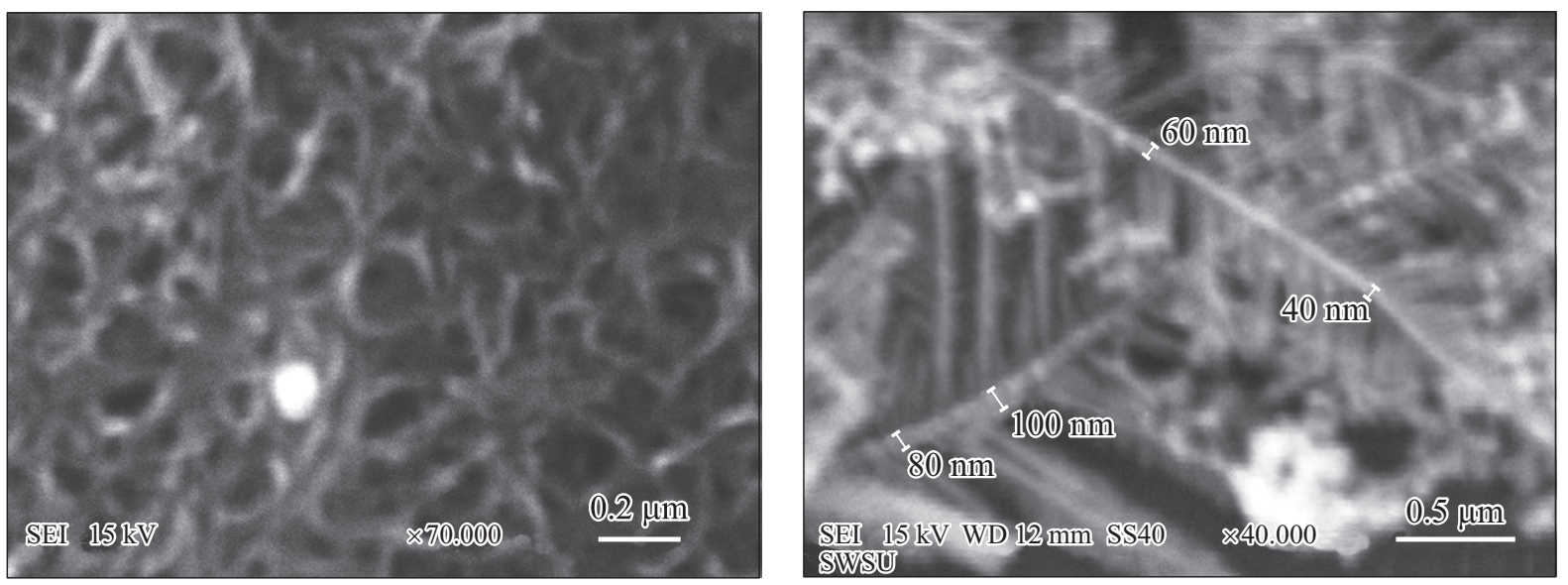

Рис. 3. Конфокальное и SEM-изображения в осадке MWCNT на ситалле: без поля $(a, c)$; линейные структуры $(b, d)$ из SDS II при $U=21 \mathrm{~V}$ и SDS I при $U=20 \mathrm{~V}$.

приведенные на SЕМ-изображении на рис. 3, $d$ при увеличении в $4 \cdot 10^{4}$ раз.

Анализ микро- и наноструктурной перестройки, происходящей в электрическом поле при испарении капли как с SDS I, так и с SDS II, (рис. 3) и (рис. 4) позволил их условно подразделить на структуры фрактальные (SF), кластерные (SC) и линейные (SL). Четких пространственно-временных границ в образовании той или иной описываемой структуры в зависимости от $U$ (в диапазоне от 10 до $50 \mathrm{~V}$ ) не установлено. Однако, как правило, первыми формировались линейные структуры и лишь через 60-90s отмечено формирование структур фрактального типа. Формирование фрактальных и линейных структур начиналось только с отрицательного электрода, а кластерные структуры возникали вблизи положительного электрода. При минимальной концентрации исследуемых растворов $(0.2 \mathrm{mg} / \mathrm{ml})$, начиная с $U=35 \mathrm{~V}$, было зарегистрировано протекание тока, который на $90 \mathrm{~s}$ от начала испарения достигал максимума - $5.5 \mathrm{~mA}$, когда высота капли уменьшалась наполовину: $h=1 / 2 h_{0}$. Затем ток плавно уменьшался почти до нуля при полном высыхании капли. Обнаружена тенденция резкого роста длины фрактальных структур при уменьшении концентрации функционализированных MWCNT. Так, их размеры составили при концентрации $0.31 \mathrm{mg} / \mathrm{ml}-6 \mu \mathrm{m}, 0.24 \mathrm{mg} / \mathrm{ml}-9 \mu \mathrm{m}$, $0.2 \mathrm{mg} / \mathrm{ml}-16 \mu \mathrm{m}$.

Минимальные размеры частиц, образующих кластерные и фрактальные структуры, оказались близкими к $20 \mathrm{~nm}$, т. е. соответствовали минимальному диаметру MWCNT (см. вставка к рис. 1). Диапазон изменения характерных размеров всех типов описываемых структур был определен по данным гранулометрического анализа AFM изображений. Типичное такое распределение приведено на вставке к рис. $4, b$ для структур из SDSI при $U=16 \mathrm{~V}$. Минимальный диаметр отдельных линейных структур из обоих растворов составлял порядка $D=70 \mathrm{~nm}$, а их длина достигала от нескольких десятков до сотен $\mu \mathrm{m}$. Расчетное число MWCNT в составе линейной структуры по толщине варьировалось 

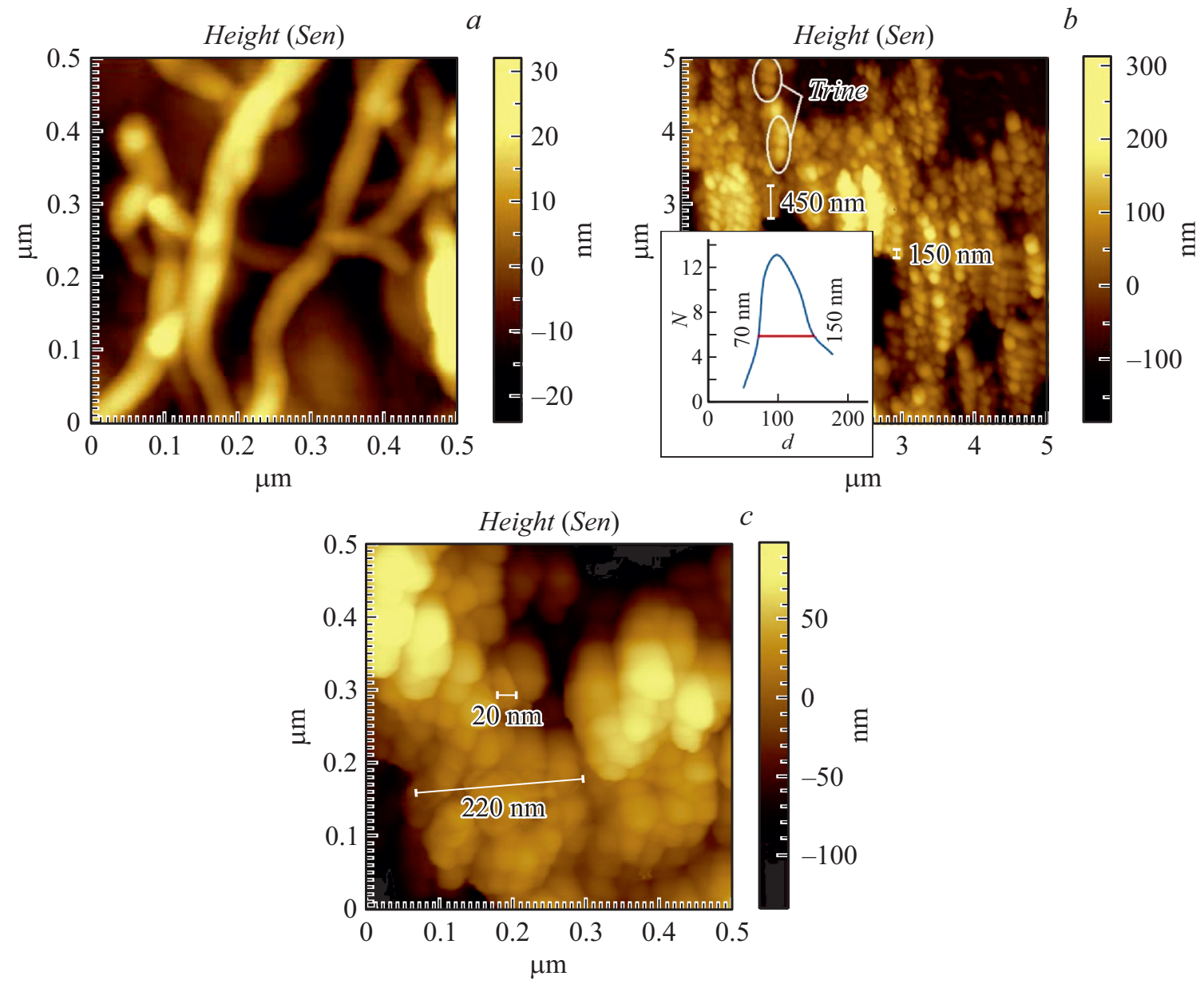

Рис. 4. АCM изображения: линейные SDS II $U=20 \mathrm{~V}(a)$ и фрактальные и линейные структуры из SDS I $U=16 \mathrm{~V}(b)$; кластерные структуры из $\operatorname{SDS}$ I $U=20 \mathrm{~V}(c)$.

от $\sim 12$ до 25 . То есть структуры линейного типа представляли собой „жгуты“. Распределения размеров структурных образований по данным AFM и SEM хорошо согласовывались (рис. $3, d$ и рис. $4, b, c$ ). B SDSI формирование фракталов и кластеров из MWCNT начиналось с $U=10 \mathrm{~V}$ и продолжалось вплоть до $U=20 \mathrm{~V}$. Структуры линейного типа возникали, начиная только с напряжения $16 \mathrm{~V}$. Более детальный анализ AFM изображений этих структур (рис. 4, $b$ ), формируемых в SDSI при $16 \mathrm{~V}$, показал, что они прямолинейны и содержали, как правило, только три отдельных агломерата, названные нами „триада“, одинакового диаметра от 70 до $150 \mathrm{~nm}$. Таким образом, наибольшая длина такой структуры доходила до $450 \mathrm{~nm}$. Подобные структуры можно видеть и на SEM-изображениях (рис. 3, $d$ ). $\mathrm{AFM}$ изображения (рис. $4, b, c)$ в электрическом поле помимо областей линейных (рис. $4, b$ ) имели участки со структурами в виде кластеров сферической формы, диаметр которых составил $\sim D 220 \mathrm{~nm}$ (рис. $4, c)$. Количество входящих в них MWCNT (с диаметром $\sim 20 \mathrm{~nm}$ ) достигало $D^{3} / d^{3} \sim 1300$.

Влияние электрического поля на процессы структурирования при испарении капли с комплексами MWCNT в
SDSI и SDSII было проанализировано по видеозаписям. Было установлено, что с ростом $U$ длина фрактальных структур - $L_{\mathrm{SF}}$ сокращалась по гиперболической зависимости - $L_{\mathrm{SF}} \sim 1 / U$ вплоть до $20 \mathrm{~nm}$, т.е. до диаметра MWCNT (рис. 5,a), а скорость их роста, наоборот, увеличивалась квадратично $V_{\mathrm{SF}} \sim U^{2}$. Величина скорости формирования фрактальных структур изменялась скачком, сохраняя постоянство в течение некоторого времени, как это видно на рис. 5, c. Так, вплоть до 15-й ходил со скоростью $V_{\mathrm{SF}}=14 \mu \mathrm{m} / \mathrm{s}$. Затем $V_{\mathrm{SF}}$ скачком увеличивалась до $33 \mu \mathrm{m} / \mathrm{s}$ и не изменялась до 22-й s, и так далее. Зависимость $V_{\mathrm{SF}}(U)$ имела также нелинейный характер (рис. 5, b). Скорость прорастания линейных структур была на 2-3 порядка выше. Фактически за время менее $1 \mathrm{~s}$ линейная структура прорастала от места возникновения до „,+“ электрода.

Анализ химических структур кластерных, фрактальных и линейных образований в электрическом поле был проведен методом рамановского рассеяния света RS с разрешением не более $500 \mathrm{~nm}$. На рис. 2 со вставками $a-$ с линией $2 D, b-$ в области радиальной дыхательной моды (RBM - radial breathing 

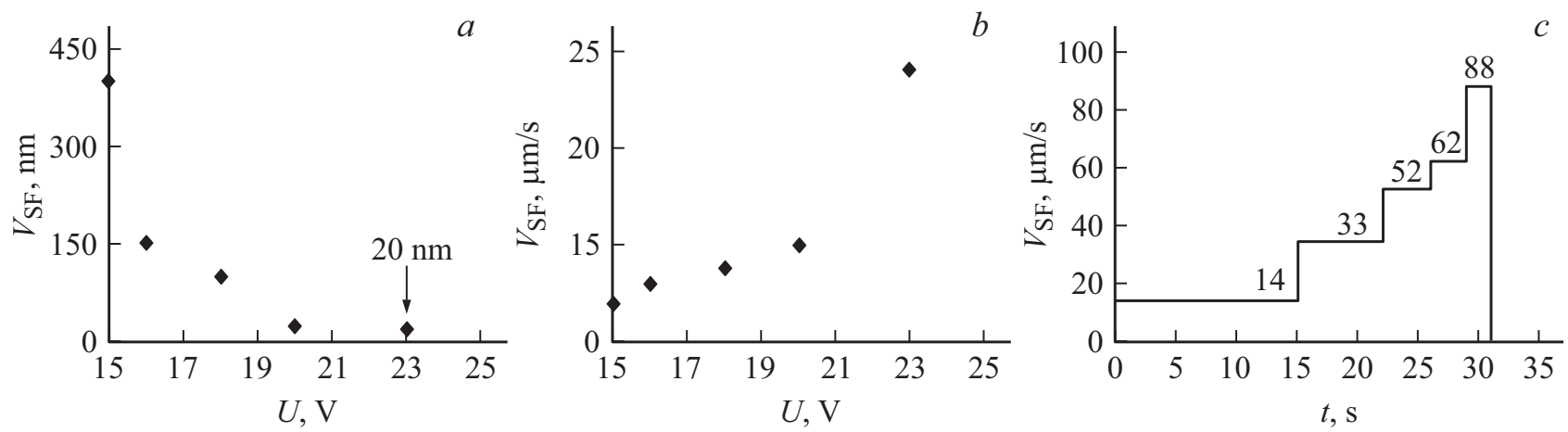

Рис. 5. Зависимость размера $(a)$, скорости роста $(b)$ для MWCNT в SD SI и динамика изменения скорости $(U=19 \mathrm{~V})$ для MWCNT в SDS II $(c)$.

Уровень дефектности, гомогенности, частоты RBM и диаметры SWCNT по RS функционализированных MWCNT в составе линейных, фрактальных и кластерных структур в электрическом поле

\begin{tabular}{l|c|c|c|c|c}
\hline Тип структуры & $\mathrm{RBM}, \mathrm{cm}^{-1}$ & $d, \mathrm{~nm}$ & $K_{\mathrm{MWCNT}}$ & $I_{2 \mathrm{D}} / I_{\mathrm{G}}$ & $L_{\mathrm{gom}}, \mathrm{nm}$ \\
\hline Линейные & 280 & 0.9 & 1.2 & 2.5 & 3.7 \\
Фрактальные & - & - & 1.3 & 1.2 & 3.4 \\
Кластерные & $193,235,272$ & $1.2,1,0.8$ & 1.4 & 1.5 & $3.1 \mathrm{M}$
\end{tabular}

mode), $c-$ в области линий $D$ и $G$ представлены спектры RS именно от указанных структур и показаны фрагменты их конфокальных изображений, обозначенные SL, SC и SF. Светлой точкой в каждом изображении отмечено положение области измерения RS.

Для всех исследуемых MWCNT интенсивности линий $I_{\mathrm{D}}, I_{2 \mathrm{D}}$ и $I_{\mathrm{G}}\left(I_{2 \mathrm{D}}-\right.$ линия двухфотонного неупругого рассеяния на вставке $a$ к рис. 2) с шириной не менее $50 \mathrm{~cm}^{-1}$ по уровню 0.5 (см. таблицу), выполнялось условие $I_{\mathrm{D}}$ и $I_{2 \mathrm{D}}>I_{\mathrm{G}}$. Это свидетельствовало о повышенном уровне их дефектности из-за остаточных поверхностных напряжений в комплексах MWCNT после интенсивной ультразвуковой обработки. Наибольшая интенсивность линий $I_{\mathrm{D}}$ и $I_{\mathrm{G}}$ отмечена в структурах типа фракталов, для кластерных она была в 4 раза ниже, а для линейных снижалась на порядок. Согласно рис. 2, при ранжировании этих структур в SDSII в следующем порядке: SL, SC и SF, так называемый коэффициент дефектности $\left(K_{\mathrm{MWCNT}}=I_{\mathrm{D}} / I_{\mathrm{G}}\right)$ возрастал, а отношение $I_{2 \mathrm{D}} / I_{\mathrm{G}}$ убывало, что согласуется с выводами [27]. В таблицу в такой же последовательности внесены убывающие расчетные значения гомогенности $\left(L_{\mathrm{g}}=4.4\left(I_{\mathrm{G}} / I_{\mathrm{D}}\right)\right)$. Обнаружена сопоставимость представленных в таблице размеров гомогенности и областей когерентности $-L_{\mathrm{c}}$ для функционализированных MWCNT. Ее расчетная величина из уравнения Дебая-Шерера $\left(L_{\mathrm{c}}=0.9 \lambda /(\beta \cos \theta)\right.$ при $\beta=0.9$ по наиболее характерной линии $(002)-$ $2 \theta=25.92$ на рентгеновской дифрактограмме) (GBC EMMA, $\left.60 \mathrm{kV}, 80 \mathrm{~mA}, \lambda=0.154 \mathrm{~nm}, \mathrm{Cu} K_{\alpha}\right)$ для этих MWCNT составила $\sim 3.5 \mathrm{~nm}$.

В окрестности линии $G$ интенсивность для линейных и фрактальных структур была наибольшая (вставка $c$ на рис. 2). Возбуждались линии $G_{1}\left(G^{+}\right)-1584$ и $1588 \mathrm{~cm}^{-1}$ и $G_{2}\left(D^{\prime}\right)-1620$ и $1606 \mathrm{~cm}^{-1}$, а также $G^{-}-\left(1526 \mathrm{~cm}^{-1}\right)$. Линии $G^{-}$и $G^{+}$соответствуют продольным колебаниям атомов во внешней оболочке относительно оси MWCNT и указывают на различие их проводимости [28]. Линия $G^{-}$характерна для MWCNT с металлической проводимостью, возникала только для структур линейных, а вот $G^{+}$, отвечающая за полупроводниковую проводимость, имела место на структурах как линейных, так и кластерных. Особенностью представленного спектра RS стало одновременное возбуждение совокупности всех возможных тангенциальных колебаний $G^{-}, G_{1}\left(G^{+}\right) G_{2}\left(D^{\prime}\right)$, что ранее, по нашим данным, не наблюдалось.

На вставке $b$ к рис. 2, с конфокальными изображениями показаны нетипичные для MWCNT колебания в низкочастотной области спектра RS - радиальные дыхательные моды (RBM), которые характерны только для SWCNT [29]. Были обнаружены линии 193, 235 и $272 \mathrm{~cm}^{-1}$ для структур кластерных, осажденных непосредственно на „+“ электроде, и для структур линейных - линия $280 \mathrm{~cm}^{-1}$. Отличительной особенностью было отсутствие этих возбуждений на структурах фрактальных. Возникновение SWCNT на концах и по оси MWCNT было подтверждено ранее методом просвечивающей электронной микроскопии высокого разрешения [30] и по данным RS [31]. Оценки диаметров наблюдаемых нами SWCNT по колебания RBM из эмпирического выражения $d_{i}=227 \mathrm{~cm}^{-1} \cdot \mathrm{nm} / \omega_{\mathrm{iRBM}}$ составили для структур линейных $\sim 0.9 \mathrm{~nm}$, а для кластерных структур на „+““ электроде их значения представлены в таблице и лежали от $\sim 0.8$ до $1.2 \mathrm{~nm}$, 
что отчасти коррелирует с большими размерами и самих этих структур из MWCNT (рис. 4, c).

По таблицам Катауры [31] с учетом энергии используемого излучения $2.33 \mathrm{eV}$ была определена хиральность обнаруженных SWCNT. Линии $280 \mathrm{~cm}^{-1}$ отвечала SWCNT с хиральностью $(8,4)$ с углом $0<\theta<30^{\circ}$. То есть структуры линейные обладали смешанной проводимостью: $1 / 3$ - металлической и $2 / 3$ - полупроводниковой. Аналогично линии 193, 235 и $272 \mathrm{~cm}^{-1}$ характеризовались хиральностями $(11,7),(12,1)$ и $(9,3)$ соответственно и также обладали смешанной проводимостью. Именно наличием металлической проводимости для этих структур обусловлены тангенциальные возбуждения $G^{-}, G_{1}\left(G^{+}\right)$и $G_{2}\left(D^{\prime}\right)$ (вставка $c$ к рис. 2) за счет колебаний смежных атомов углерода в решетке трубок вдоль и перпендикулярно их оси при неизменной поляризации излучения. Интенсивности линий $I_{\mathrm{G}}^{-}, I_{\mathrm{G}}^{+}$ и $I_{\mathrm{G} 2}$ (вставка $c$ на рис. 2) удовлетворяли условиям: $I_{\mathrm{G}}^{-} \ll I_{\mathrm{G}}^{+}$и $I_{\mathrm{G} 2}<I_{\mathrm{G}}^{+}$, что также указывало на наличие полупроводникового типа проводимости, а возбуждение линии $G^{-}$на частично металлический тип их проводимости [28].

\section{Обсуждение результатов}

Как уже отмечалось, в настоящее время активно изучаются материалы, содержащие включения CNT, при электрических и других воздействиях [4,9,12-16], также как и процессы структурирования самих MWCNT и SWCNT в этих условиях [10,11,24,25,32-35]. Отметим, что в таких исследованиях важной является пространственно-временная взаимосвязь структурных изменений CNT с внешними воздействиями. Обобщенно, описание процесса структурирования происходило по сценарию, имеющему аналогию с описанным ранее в [7,24,35]. Микрочастицы из агломерированных функционализированных MWCNT или их наноразмерных частей объединялись в структуры, кластерные на поверхности электрода „+““, одновременно с этим начинали расти структуры фрактальные и линейные с электрода „-“. То есть фактически имела место их самоорганизация по стандартной схеме „агрегаты “ до $300 \mathrm{~nm}-$ кластеры (SC) $\Rightarrow$ „жесткие“ агломераты микронных размеров - фракталы $(\mathrm{SF}) \Rightarrow$ „мягкие“ агломераты до десятков $\mu \mathrm{m}$ - линейные структуры (SL). Источниками энергии для формирования этих самоорганизованных структур, очевидно, могли выступать энергия электрического поля и тепловая энергия, выделяемая в процессе испарения капли с растворенными MWCNT.

Диффузионная модель испарения капли Дж.К. Максвелла, которая была ранее применена [24,25] для анализа процессов структурирования анионных и катионных микро- и нановключений в минеральных водах, применительно к исследуемым в электрическом поле растворам SDSI и SDSII, должна быть дополнена силами электрической природы. Число Рэлея $\mathrm{Mg}=D_{\mathrm{dr}} \Delta T(\alpha \eta)^{-1} d \sigma / d T$, определяемое динамической вязкостью $\eta$, температуропроводностью $\alpha$ и поверхностным натяжением воды $\sigma$, с учетом крайне малого изменения температур $\Delta T \sim 10^{-1} \mathrm{C}$ между поверхностью капли и подложкой при испарении, удовлетворяет условию $\mathrm{Mg} \ll 1$. То есть конвективные движения исключены. Однако на радиально-осевое распределение MWCNT, зависевшее ранее только от гидродинамических потоков (Рэлея-Бенара, Марангони и компенсационных, согласно [26]), накладывалось сильное влияние электрических сил. В соответствии с опытными данными [14,32] в результате достигается разделение CNT с учетом характерных для них металлической и полупроводникой проводимостей, наличие которых в нашем случае было также подтверждено спектрами RS (рис. 2). В отличие от углеродных образцов в [24] функционализированные MWCNT [25,36] в электрическом поле дополнительно отрицательно поляризовались с образованием „эффективного дипольного момента“"

С учетом того что при RS интенсивность рассеянного излучения $(I)$ под углом $\psi$ к направлению падающего поляризованного $\left(I_{0}\right)$ описывается законом Малюса: $I=I_{0} \cos ^{4}(\psi)$, для, как правило, разупорядоченных CNT рассеяние света вызвано характерными для них колебаниями - тангенциальным $I_{\mathrm{G}}$ и продольным $I_{\mathrm{D}}$. Картина изменяется при взаимодействии с поляризованными в электрическом поле MWCNT. Например, для G-моды можно записать распределение $N$ нанотрубок, ориентирующихся под углом $\chi-N(\chi)$ относительно вертикальной $-I_{\perp}$ и горизонтальной $-I_{\|}$поляризаций падающего света [31]:

$$
\begin{gathered}
I_{\perp}=I_{0} \int_{-\pi / 2}^{\pi / 2} N(\chi) \cos (\psi-\chi)^{4} d \chi, \\
I_{\|}=I_{0} \int_{-\pi / 2}^{\pi / 2} N(\chi) \cos ^{2}(\psi-\chi) \sin ^{2}(\psi-\chi) d \chi .
\end{gathered}
$$

В нашем случае поляризация падающего излучения $I_{0}$ всегда была одинаковой. Поэтому $I_{\perp}$ и $I_{\|}$могли изменяться только за счет ориентации самой MWCNT, что обусловливало появление колебаний относительно ее продольной оси: $\alpha_{\perp}-$ с поперечной и $\alpha_{\|}-$ продольной поляризациями. Эти возбуждения в области $\mathrm{RBM}$ для трубок типа „зигзаг“ $-(n, 0)$ будут больше, чем для трубок типа „кресло“ - $(n, n)$. Поперечная поляризация оказывается линейно зависимой: $\alpha_{\perp} \sim l$, тогда как продольная $\alpha_{\|} \sim l^{2}$ [35]. Хиральности обнаруженных SWCNT были смешанного типа $(n, m)$ : линейные структуры - $(8,4)$, кластерные структуры $(9,3),(11,7)$ и $(12,1)$. Однако интенсивность спектра в области RBM для линейных структур была выше, чем у кластерных (ср. рис. 2, вставка $b$ ). Косвенно это указывало на большую роль металлической проводимости, что подтверждалось более явной ориентацией линейных структур вдоль поля, а также высокой 
скоростью роста $\geq 1 \cdot 10^{3} \mu \mathrm{m} / \mathrm{s}$, которая в десятки раз превышала скорость роста вдоль поля фрактальных структур $-25 \mu \mathrm{m} / \mathrm{s}$ (рис. $5, b$ ). Очевидно, что энергия структурирования MWCNT электрическим полем была выше энергии их тепловых колебаний $(1 / 2) k T$. Таким образом, основное влияние на поведение MWCNT в электрическом поле могли оказывать как их поляризации $\alpha_{\perp}$ и $\alpha_{\|}$, так и их собственная проводимость, т. е. их хиральность. Поперечная поляризация оказывается линейно зависимой: $\alpha_{\perp} \sim l$, тогда как продольная $\alpha_{\|} \sim l^{2}$, а возникающий в поле крутящий момент поляризации за счет $\alpha_{\perp}$ и $\alpha_{\|}$, согласно [35], составлял

$$
M=(1 / 2) E^{2}\left(\alpha_{\|}-\alpha_{\perp}\right) \sin 2 \theta,
$$

где угол $\theta$ соответствует ориентации MWCNT относительно $E$. Под действием $M$ трубки с большим $l$ образовывали „жгуты“ большого размера как по диаметру, так и по длине вплоть до межэлектродного расстояния (рис. $3, b, d$ ). Отмеченное выше протекание тока между электродами „-““ и „,+“ с максимумом $5.5 \mathrm{~mA}$ на $90 \mathrm{~s}$, когда в капле с MWCNT зафиксировано образование линейных структур, может указывать на включение механизма собственной проводимости, характерной для SWCNT.

В соответствии с (3) короткие трубки (в пределе с $l \sim d$, когда $l / d \sim \alpha_{\|} / \alpha_{\perp}$, т. е. $\alpha_{\|} \sim \alpha_{\perp}$ крутящий момент $M=0$ ) структурировались по-другому, образуя кластерные структуры (рис. 4, c). Таким образом, MWCNT c малой длиной теряли способность к направленному движению от „-“ к „+““. В результате формировались кластерные структуры, которые распределялись в капле и особенно вблизи поверхности „+““ электрода (вставка $b$ с конфокальными изображениями SC на рис. 2), что соответствовало отсутствию в них „эффективного дипольного момента“. Форма этих структур была сферической с размерами (свыше $220 \mathrm{~nm}$ ) (рис. $4, c)$.

Наблюдался еще один тип упорядочения в электрическом поле с образованием типичных фрактальных структур (рис. 2, с и рис. 4, b), формирование которых происходило и без поля (рис. $3, a$ ). В таких структурах колебания в области RBM отсутствовали, что указывало на отсутствие в них SWCNT. Рост таких структур в поле, как показал анализ видеозаписей, происходил только в направлении от „-“ к „++“. Изменения размеров и скорости роста таких структур приведены на рис. 5. По данным RS (см. таблицу), они обладали наибольшей

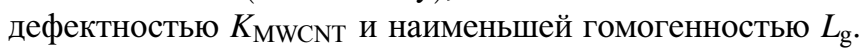

Верхняя и нижняя границы, в которых может реализоваться сценарий устойчивого фракталообразования, определяется начальными и конечными размерами капли после ее испарения $\left(h_{\max }=h_{0}=500 \mathrm{~nm} \quad\right.$ и $\left.h_{\min }=120 \mathrm{~nm}\right)$. Скорость агломерации частиц из MWCNT должна подчиняться уравнению Фукса $\partial n / \partial t=-8 \pi R D n^{2} \exp \left(-E_{\mathrm{ag}} / k T\right)$. Здесь $n-$ концентрация не агломерированных частиц, $E_{\mathrm{ag}}-$ высота энергетического барьера агломерации, убывающая с уменьшением высоты капли $h, R$ - их радиус, $D-$ коэффициент диффузии, который можно принять $\sim 1 / R$. Согласно этому уравнению, можно заключить, что если в начале структуры фрактальные растут преимущественно из MWCNT небольших размеров, начиная с $20 \mathrm{~nm}$, с наибольшей $D$, то по мере уменьшения $n$ происходит агломерация уже MWCNT больших размеров (150 nm и более). Характерной особенностью структурирования жгутов из MWCNT является их объединение (коагуляция) в устойчивые тройные структуры - „триады“, как это видно из (рис. $4, b$ ). Стабильное существование таких триад, возможно, обусловлено тем, что только в такой конфигурации центральный комплекс MWCNT становится электронейтральным. Триада выступает далее как устойчивая структура с субмикронными размерами $450 \mathrm{~nm}$, с объединенным „эффективным дипольным моментом“, что подтверждается ее четкой ориентацией вдоль поля (рис. $4, b)$.

Анализ микрофотографий (рис. 3 и 4) и видеоизображений в еще не испарившейся капле, когда формируются все описываемые структуры, свидетельствует о доминирующем влиянии сил электрической природы: кулоновской $\left(F_{\mathrm{q}}=q E\right.$, где $q$ - заряд ,эффективного дипольного момента“") и диэлектрофоретической $-F_{\mathrm{def}}[14,32]$. В наших условиях $(E=\mathrm{const}) F_{\mathrm{def}}$, пропорциональная градиенту квадрата напряженности электрического поля $-\nabla E^{2}$, будет отсутствовать. Поэтому на формирование линейных и фрактальных структур основное воздействие будет оказывать $F_{\mathrm{q}}$. В процессе изготовления SDS I и SDS II после кипячения в растворе $\mathrm{H}_{2} \mathrm{SO}_{4}$ и $\mathrm{HNO}_{3}$, удаляющего наночастицы Со из MWCNT, возникновение их „эффективного дипольного момента“ может быть обусловлено только присоединением к ней карбоксильной группы $\mathrm{COOH}^{-}$с равномерным распределением вокруг нее по всей длине $l$. В результате трубка оказывается внутри отрицательно заряженной оболочки. Величина этого заряда зависит от размеров MWCNT $(l, d)$, характерных размеров между двумя смежными атомами в гексагоне, участвующими в образовании связи с карбоксильной группой $\mathrm{COOH}^{-}$и равна 120lde, где $e$ - заряд электрона. Именно за счет этого заряда возникает поляризация и движение нанокластеров из MWCNT с образованием структур линейных и фрактальных в направлении от „-“ к к „+““ в поле с напряженностью $E$. Сила, действующая на элемент цилиндрической поверхности $\Delta S_{\mathrm{q}}=\pi d \Delta l$, перпендикулярный к полю, равна $\Delta F_{\mathrm{q}}=(1 / 2) \varepsilon_{0} \varepsilon E^{2} \pi d \Delta l$, где $\varepsilon_{0}$ и $\varepsilon$ - диэлектрическая постоянная MWCNT и диэлектрическая проницаемость воды. Диэлектрическая постоянная MWCNT в постоянном поле зависит от их хиральности, и для трубок с полупроводникой проводимостью будет существенно ниже $\varepsilon$ для воды. Сила, действующая на трубку по всей высоте, $F_{\mathrm{q}}=\int(1 / 2) \varepsilon_{0} \varepsilon \pi d E^{2} d l=(1 / 2) \varepsilon_{0} \varepsilon E^{2} \pi d l$. Таким образом, $F_{\mathrm{q}}$ будет возрастать при увеличении как длины, так и диаметра MWCNT. Модельные расчеты влияния длины 
и диаметра на такую же силу при росте MWCNT на массивном нанокатализаторе в постоянном электрическом поле дали аналогичные результаты [37]. Там же показано, что роль силы $F_{\mathrm{q}}$ возрастает в зависимости от числа MWCNT и уменьшения расстояния между ними. Характерно, что это согласуется с реально наблюдаемым нами объединением трубок в нанокластеры, из которых в дальнейшем строятся указанные линейные и фрактальные структуры.

Если исходить из того, что для построения такой структуры между электродами с расстоянием $L$ потребуется $N$ комплексов из MWCNT с диаметром $d(L=N d)$, то, очевидно, зависимость размеров фрактальных структур от прикладываемого напряжения $L_{\mathrm{SF}}=f(E)$ будет гиперболической $\sim 1 / E$. В пределе $L_{\mathrm{SF}} \rightarrow d=20 \mathrm{~nm}$ (рис. 5, a).

Если исходить из того, что при фрактальном структурировании наиболее сильное влияние оказывает электрическое поле, уравнение движения нанокластеров можно упрощенно записать в виде $m d v / d t=F_{\mathrm{q}}$. Тогда c учетом конечности времени испарения капли как верхнего предела интегрирования может быть получена приближенная зависимость $V(U)$ :

$$
V(E) \sim(\rho)^{-1} \varepsilon_{0} \varepsilon E^{2} \int d t=V_{0}+B E^{2},
$$

где $V_{0}$ - начальная скорость, величина которой зависит от диффузии MWCNT в изучаемом растворе, $B$ постоянная, учитывающая $\rho-$ плотность MWCNT $\left(\sim 2.2 \mathrm{~g} / \mathrm{cm}^{2}\right)$ и диэлектрическую проницаемость воды после SDS. Квадратичная зависимость (4) качественно согласуется с опытными данными (рис. 5, b).

Скорость образования фрактальных структур фактически изменялась скачком, оставаясь постоянной в течение некоторого времени (рис. 5, c). Следует учесть, что в процессе испарения капли изменения концентрации $(C)$ обусловлены двумя прямо противоположными процессами: как ростом $C$ по мере уменьшения высоты капли $(d C / d t)_{h}>0$, так и снижением из-за формирования этих структур $-(d C / d t)_{\mathrm{C}}<0$. Устойчивый рост фракталов, очевидно, происходит при $(d C / d t)_{\mathrm{C}}=(d C / d t)_{h}$, когда скорость роста структур неизменна $\left(U_{\mathrm{SF}}=\mathrm{const}\right)$. При условии $(d C / d t)_{\mathrm{C}}<(d C / d t)_{h}$ возникает скачок скорости, т. е. включается флуктуационный механизм. Согласно рис. $5, c$, длительности интервалов $\tau_{\mathrm{SF}}$ на зависимости $V_{\mathrm{SF}}(t)$ сокращаются, а величины скачков $\Delta V_{\mathrm{SF}}(t)$ растут. Можно сказать, что условие стационарного роста фрактальных структур $(d C / d t)_{\mathrm{C}}=(d C / d t)_{\mathrm{h}}$ обеспечивается как линейным уменьшением $C$ по мере испарения капли, так и нелинейным ростом кулоновской силы при ее приближении к электроду.

\section{Заключение}

Коллоидная система из функционализированных многостенных углеродных трубок после продолжительного ультразвукового диспергирования получила высокую стабильность, сохраняющуюся в течение уже четырех лет. В результате самосборки и/или самоорганизации многостенных нанотрубок в такой коллоидной системе в электрическом поле формируются структуры: линейные, фрактальные и кластерные, которые по данным комбинационного (рамановского) рассеяния света существенно отличаются по химической структуре. Внутри многостенных нанотрубок обнаружены одностенные трубки с металлической и полупроводникой проводимостями, которые участвуют в образовании линейных и кластерных структур. Рост фрактальных структур обусловлен ,эффективным дипольным моментом“ ${ }^{6}$ и носит флуктуационный характер в зависимости от изменения концентрации коллоидной системы. Установлено, что в постоянном электрическом поле размеры фрактальных структур уменьшаются как $1 / E$, скорость их роста возрастает как $E^{2}$, сохраняя постоянство в течение интервала $\tau_{\mathrm{SF}}$ при выполнении условия.

\section{Финансирование работы}

Работа выполнена при финансовой поддержке Минобрнауки России в рамках задания № 16.2814.2017/ПЧ.

\section{Конфликт интересов}

Авторы заявляют, что у них нет конфликта интересов.

\section{Список литературы}

[1] Yuchi Che, Haitian Chen, Hui Gui, Jia Liu, Bilu Liu, Chongwu Zhou // Semicond. Sci. Technol. 2014. Vol. 29. P. 073001(17).

[2] Aikawa Sh., Kim S., Thurakitseree Th. etal. // Appl. Phys. Lett. 2018. Vol. 112. P. 013501(5).

[3] Le V.Th., Ngo C.L., Le Q.Tr. etal. // Advances In Natural Sciences: Nanoscience and Nanotechnology. 2013. Vol. 4. P. $035017(5 \mathrm{p})$.

[4] Birylin Yu., Kurdybaylo D., Shamanin V. etal. // Fullerenes, Nanotubes and Carbon Nanostructures. 2008. Vol. 16. N 586. P. 529-633.

[5] Reinert L., Zeiger M., Suarez S. etal. // Royal Society of Chemistry Adv. 2015. Vol. 5. P. 95149-95159.

[6] Hosseini H., Mahdavi H. // Appl. Organometal Chem. 2018. Vol. 4294. P. 1-8. https://doi.org/10.1002/aoc.4294

[7] Воробьева А.И. // УФН. 2010. № 3. С. 265-288.

[8] Singh D.K., Iyer P.K., Giri P.K. // Diamond \& Related Materials. 2010. N 19. P. 1281-1288.

[9] Lehman J.H., Terrones M., Mansfield El. etal. // Carbon. 2011. Vol. 49. P. 2581-2602.

[10] Li J., Zhang Q., Peng N. etal. // Appl. Phys. Lett. 2005. Vol. 86. P. 153116(4).

[11] Давлеткильдеев Н.А., Соколов Д.В., Болотов В.В., Лобов И.А. // Письма в ЖТФ. 2017. Т. 43. Вып. 4. С. 47-55.

[12] Vul' A., Reich K., Eidelman Ev. etal. // Advan. Sci. Lett. 2010. Vol. 3. N 2. P. 110-116.

[13] Sameera I., Bhatia R., Prasad V. etal. // J. Appl. Phys. 2012. Vol. 111. P. 044307(6). 
[14] Banerjee S., White B.E., Huang L. etal. // J. Vac. Sci. Technol. 2006. Nov/Dec. B. Vol. 24. N 6. P. 3173-3178.

[15] Агеев О.А., Блинов Ю.Ф., Ильина М.В., Ильин О.И., Смирнов В.А., Цуканова О.Г. // ФТТ. 2016. Т. 58. Вып. 2. C. 301-306.

[16] Горшков М.В., Москаленко А.С., Павельев В.C. идр. // Информационные технологии и нанотехнологии. Компьютерная оптика и нанофотоника. 2017. C. $328-332$.

[17] Бельский М.Д., Бочаров Г.С., Елецкий А.В., Sommerer T.J. // ЖТФ. 2010. Т. 80. Вып. 2. С. 130-137.

[18] Fitnat S., Naveed Ah., Saqib H. etal. // Appl. Sci. 2018. Vol. 8. P. 395. DOI: 10.3390/app8030395

[19] Yun D.-J., Jeong Y.J., Ra H. etal. // Organic Electron. 2018. Vol. 52.

[20] Saikiran V., Bazylewski P., Sameera I. etal. // Appl. Surf. Sci. 2018. Vol. 439. P. 823-832.

[21] Huang Y.Y., Terentjev Eu.M. etal. // Polymers. 2012. Vol. 4. P. 275-295.

[22] Sadia S., Nadeem Iq., Asghari M. etal. // J. Physics: Conference Series. 2013. Vol. 439. P. 0120(24).

[23] Datsyuk V., Kalyva M., Papagelis K. etal. // Carbon. 2008. N 46. P. 833-840.

[24] Кузьменко А.П., Чан Ньен Аунг, Родионов В.В. // ЖТФ. 2015. T. 85. Вып. 6. C. 118-125. [Kuz'menko A.P., Chan N.A., Rodionov V.V. // Tech. Phys. 2015. Vol. 60. N 6. P. 903-910.]

[25] Кузьменко А.П., Тет Пьо Наинг, Мьо Мин Тан идр. // Известия Юго-Западного гос. ун-та. Серия техника и технологии. 2015. № 3(16). С. 39-50.

[26] Andreeva L.V., Koshkin A.V., Lebedev-Stepanov P.V. etal. // Colloid. Surf. A: Physicochem. Eng. Aspects. 2007. N 300. P. 300-306.

[27] Kuznetsov V.L., Bokova-Sirosh S.N., Moseenkov I.S. etal. // Phys. Stat. Sol. B. 2014. 251. N 12. P. 2444-2450.

[28] Telg H., Fouquet M., Maultzsch J. etal. // Phys. Stat. Sol. 2008. Vol. 245. N 10. P. 2189-2192.

[29] Christian Thomsen Ch., Reich St. // Springer-Verlag Berlin Heidelberg-2007: M. Cardona, R. Merlin (Eds.): Light Scattering in Solid IX, Topics Appl. Phys. 108. P. 115-232.

[30] Qin L.-Ch., Zhao X., Hirahara K. etal. // Nature. 2000. N 408. P. 50-51.

[31] Dresselhaus M.S., Jorio A., Hofmann M. et al. // Nano Letters. 2010. N 10. P. 751-758.

[32] Krupke R., Hennrich F., Lohneysen H. etal. // Science. 2003. N 301. P. 344-347.

[33] Rodriguez R.D., Marius T., Sascha H. etal. // Nano. Res. Lett. 2012. Vol. 7. P. 682.

[34] Li J., He Y., Han Y. etal. // Nano Lett. 2012. N 12. P. 4095-4101.

[35] Ma Sh.-J., Guo W.-L. // Chin. Phys. Lett. 2008. Vol. 25. N 1. P. 270-273.

[36] Кузьменко А.П., Наинг Т.П., Кузько А.Е. идр. // Известия вуз. Материалы электронной техники. 2016. Т. 19. № 4. С. 269-276.

[37] Bao Q., Zhang H., Pan Ch. // Computational Mater. Sci. 2007. Vol. 39. P. 616-626. 